\title{
Commentary: Response to commentary by Bilgin and Wolke (2021)
}

Robin S. S. Kramer and Abi M. B. Davis

School of Psychology, University of Lincoln, Lincoln, UK

Correspondence: Robin S. S. Kramer, School of Psychology, University of Lincoln, Brayford Pool, Lincoln LN6 7TS, UK.

E-mail: remarknibor@gmail.com

Telephone: +44 (0) 1522835806

Fax: $+44(0) 1522886489$

This is a pre-print with copyright owned by the authors.

Running title: Commentary on Bilgin and Wolke (2021) 


\begin{abstract}
Recently in this journal, Bilgin and Wolke (2020) published a paper which showed no association between leaving infants to 'cry it out' and infant-mother attachment. In our commentary (Davis \& Kramer, 2021), we argued that the study was underpowered and, as such, could not be considered a conclusive demonstration that 'cry it out' had no adverse effects on attachment. The authors (Bilgin \& Wolke, 2021) responded by claiming that, in combination with previous research, there was now 'overwhelming evidence that letting a baby to cry it out a few times or even often has no adverse effects for infant-mother attachment formation during infancy' (p. 2). Here, we show that their study was indeed underpowered and argue that there is, as yet, no compelling evidence that 'cry it out' is not harmful for attachment. Therefore, this technique should not be utilised by parents until more conclusive evidence has been provided.
\end{abstract}

Keywords: attachment; cry it out; infancy 


\section{Introduction}

In their recent article, Bilgin and Wolke (2020) investigated the potential impact of leaving infants to 'cry it out' on subsequent attachment between mothers and children. The authors' analyses showed no association between leaving infants to cry it out and infant-mother attachment at 18 months, leading the authors to state that there were 'no adverse effects on attachment'(title) and 'no adverse impacts of leaving infants to cry it out in the first 6 months' (abstract). In our commentary that followed (Davis \& Kramer, 2021), we argued that these conclusions were overstated and potentially detrimental, given the possibility to mislead the general public into thinking that the use of 'cry it out' would not cause any harm to their infants. The authors (Bilgin \& Wolke, 2021, p. 1) responded by suggesting we were mistaken, instead highlighting their conclusion that 'contemporary practice by some parents to occasionally or often 'leaving infant to cry it out' during the first 6 months was not associated with adverse behavioural development and attachment at 18 months'. Although perhaps less grand in its tone, we read this as the equivalent in meaning when compared with the wording already mentioned. Further, if the findings should be interpreted in a more nuanced manner then oversimplified and overstated phrases should be avoided in the title or abstract of the article - it is the authors' responsibility to ensure that their findings cannot be misinterpreted. Our argument that their results are indeed overstated is primarily based on power and sample size, and so we discuss this issue first.

\section{Power and sample size}

In the original article, the authors claim that there was 'sufficient statistical power' for their analytical strategy (Bilgin \& Wolke, 2020, p. 1185) although this power analysis was not provided. In their recent response (Bilgin \& Wolke, 2021), they again failed to supply a power analysis but instead presented a simplified scenario based on known frequencies taken 
from their sample. Here, the authors described a situation in which 68 mothers never use 'cry it out' and $75 \%$ of their babies enjoy a secure attachment. Alongside these, 110 mothers employ 'cry it out' a few times or often, with only $55 \%$ of their babies being securely attached. In order to achieve $80 \%$ power to detect this $20 \%$ difference at an alpha level of .05, the authors reported needing an overall sample size of 183. Although their sample consisted of 178 dyads, they interpreted this as 'just sufficient' (p. 2). Even if we accepted this calculation and their interpretation, there are a number of problems when comparing this hypothetical situation with the analyses that were actually carried out.

First, our calculations place the required sample size at 194 (G*Power) or 200 (R programming language), making the claim of 'just sufficient' to be an even greater stretch of this minimum boundary. Second, this value is only true if the amount of variability in the main predictor (using 'cry it out') has zero association with the covariates in the analysis, which is almost certainly not the case. For instance, the association between income and the use of 'cry it out' has recently been established (Giesbrecht et al., 2020). For non-zero associations, the required sample size will increase. Third, the sampling ratio considered $(110 / 178=0.62)$ was true only for infants at 3 months old. For the analysis of 6 -month-olds, for example, this ratio $(120 / 178=0.67)$ would result in a required sample size of 209 . Fourth, the authors simplified by combining 'a few times' and 'often' into one category for their power calculation. However, these represented two separate groups of mothers in their original analyses. Since each group was compared with mothers who 'never' used 'cry it out', each comparison would also need to be sufficiently powered. For example, if 55\% of 'often' babies were securely attached then comparing only these with the 'never' category would require a sample size of 232 (due to the new sampling ratio: $23 / 91=0.25$ ). Of course, this does not include the third group of mothers ('a few times') in their sample and the number of these required to carry out sufficiently powered analyses with their data. 
Taken together, what conclusions can we draw regarding the original study? Even if we accepted the authors' oversimplified power analysis, they themselves presumably acknowledge that their logistic regression analyses, when excluding the 73 very preterm/very low birthweight infants, was markedly underpowered. (Interestingly, income differed between full-term and very preterm dyads, which, as noted earlier, is also associated with the use of 'cry it out'.) Next, for the reasons outlined above (associations with covariates, the use of three categories rather than two, etc.), there exists no situation in which the original sample size would have provided sufficient power to detect a change of $20 \%$. Finally, this $20 \%$ effect size was suggested as clinically significant, but we argue that smaller effects would also be highly influential, and hence important, within the population. For instance, if using 'cry it out' resulted in only a $10 \%$ increase in the prevalence of insecure and disorganised attachment styles then we would still challenge its use, given the well-established negative effects of these attachment styles across a variety of domains, including mental health, aggression, and behaviours more generally (e.g., Fearon et al., 2010). Returning to the scenario presented by the authors, if 68 mothers never use 'cry it out' (70\% secure) and 110 mothers use it a few times or often $(60 \%$ secure $)$, the proposed sampling ratio $(0.62)$ would result in a required sample size of 766 in order to demonstrate sufficient power. As the authors acknowledged (Bilgin \& Wolke, 2021, p. 2), their study was 'underpowered to detect smaller overall differences', which is not to say that these differences are not important.

\section{Previous literature}

Although we originally argued (Davis \& Kramer, 2021) that attachment theory strongly suggests the importance of mothers responding promptly to their infants rather than leaving them to cry it out, the authors (Bilgin \& Wolke, 2021) correctly highlighted the lack of evidence supporting this idea. They summarised the studies of relevance, noting that only one 
demonstrated the detrimental nature of 'cry it out' on attachment security (Bell \& Ainsworth, 1972) while the remaining three found no such association (Bilgin \& Wolke, 2020;

Giesbrecht et al., 2020; van IJzendoorn \& Hubbard, 2000). However, in contrast with the views of Bilgin and Wolke (2021), we argue that none of these studies were sufficiently powered to detect any negative effects of 'cry it out'. Of the three additional studies mentioned, it seems clear that sample sizes of 26 (Bell \& Ainsworth, 1972) and 50 (van IJzendoorn \& Hubbard, 2000) were underpowered. In addition, given that the above discussion regarding power also applies to the study by Giesbrecht and colleagues (2020), since they employ a similar logistic regression strategy but with only 137 dyads, their analyses should also be considered underpowered. Taken together, at best, there have so far been no compelling studies of sufficient statistical power to inform either way regarding the (lack of) adverse effects on attachment due to 'cry it out'. Indeed, we strongly disagree with the authors' assertion that 'there is overwhelming evidence that letting a baby to cry it out a few times or even often has no adverse effects for infant-mother attachment formation during infancy' (Bilgin \& Wolke, 2021, p. 2). As ever, it is especially important for psychologists to remember that simply failing to reject the null hypothesis, as we previously noted (Davis \& Kramer, 2021), is not convincing evidence that no effect is present.

\section{Amount of crying}

Bilgin and Wolke (2021) argued that evidence suggests the early use of 'cry it out' decreases the amount of crying seen in later infanthood (Bilgin \& Wolke, 2020; Giesbrecht et al., 2020). We do not disagree. It may well be the case that leaving infants to cry it out will reduce the amount of crying subsequently, but we do not see how this speaks to the quality of the infant-mother attachment. One could easily hypothesise that infants have learned to a) cry less since their mothers do not respond; while b) feeling less secure in their attachments 
because their mothers are unresponsive. This result of 'cry it out' should not obscure what is, in our opinion, the more important concern - that readers will be (mis)led to believe that leaving their infants to cry it out will not affect their attachment.

\section{Our recommendations}

Although we appear to be at an impasse regarding our interpretation of the evidence in comparison with the original authors (Bilgin \& Wolke, 2020), we have demonstrated here that the most important conclusion to be drawn is that there is simply insufficient evidence to draw one. Focussing on Bilgin and Wolke's (2020) study in particular, we agree that their results provide no support for the hypothesis that 'cry it out' is detrimental for infant-mother attachment. However, their underpowered study failed to demonstrate a lack of an association either. As well as the incorporation of larger sample sizes, future studies should consider utilising Bayesian approaches in order to provide a better understanding of how much support the data provide for the null hypothesis - in this case, that 'cry it out' has no adverse effects. Until such studies are carried out, along with the corresponding meta-analyses on this topic (as noted in our original commentary), we once again hope that interested parents do not decide to utilise 'cry it out' on the basis of the few studies by Bilgin and Wolke (2020) and others discussed here.

\section{References}

Bell, S. M. \& Ainsworth, M. D. S. (1972). Infant crying and maternal responsiveness. Child Development, 43, 1171-1190.

Bilgin, A., \& Wolke, D. (2020). Parental use of 'cry it out' in infants: No adverse effects on attachment and behavioural development at 18 months. Journal of Child Psychology and Psychiatry, 61(11), 1184-1193. 
Bilgin, A., \& Wolke, D. (2021). Response to commentary by Davis and Kramer (2021). Journal of Child Psychology and Psychiatry. Advance online publication.

Davis, A. M. B., \& Kramer, R. S. S. (2021). Does 'cry it out' really have no adverse effects on attachment? Reflections on Bilgin and Wolke (2020). Journal of Child Psychology and Psychiatry. Advance online publication.

Fearon, R. P., Bakermans-Kranenburg, M. J., van IJzendoorn, M. H., Lapsley, A. -M., \& Roisman, G. I. (2010). The significance of insecure attachment and disorganization in the development of children's externalizing behavior: A meta-analytic study. Child Development, 81(2), 435-456.

Giesbrecht, G. F., Letourneau, N., Campbell, T., Hart, M., Thomas, J. C., Tomfohr-Madsen, L., \& APrON Study Team. (2020). Parental use of "cry out" in a community sample during the first year of infant life. Journal of Developmental \& Behavioral Pediatrics, 41(5), 379-387.

van IJzendoorn, M. H., \& Hubbard, F. O. A. (2000). Are infant crying and maternal responsiveness during the first year related to infant-mother attachment at 15 months? Attachment \& Human Development, 2(3), 371-391. 